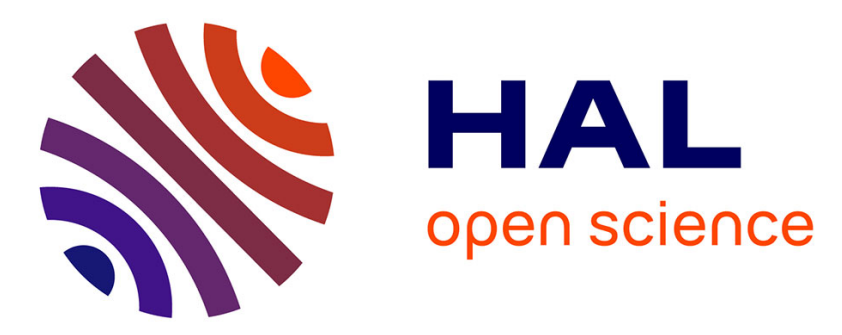

\title{
On-Demand Source Routing with Reduced Packets Protocol in Mobile Ad-hoc Networks
}

Mawloud Omar, Sabrine Hedjaz, Souhila Rebouh, Katia Aouchar, Bournane Abbache, Abdelkamel Tari

\section{- To cite this version:}

Mawloud Omar, Sabrine Hedjaz, Souhila Rebouh, Katia Aouchar, Bournane Abbache, et al.. OnDemand Source Routing with Reduced Packets Protocol in Mobile Ad-hoc Networks. AEÜ - International Journal of Electronics and Communications / Archiv für Elektronik und Übertragungstechnik, 2015. hal-03033771

\section{HAL Id: hal-03033771 https://hal.science/hal-03033771}

Submitted on 1 Dec 2020

HAL is a multi-disciplinary open access archive for the deposit and dissemination of scientific research documents, whether they are published or not. The documents may come from teaching and research institutions in France or abroad, or from public or private research centers.
L'archive ouverte pluridisciplinaire HAL, est destinée au dépôt et à la diffusion de documents scientifiques de niveau recherche, publiés ou non, émanant des établissements d'enseignement et de recherche français ou étrangers, des laboratoires publics ou privés. 


\title{
On-Demand Source Routing with Reduced Packets Protocol in Mobile Ad-hoc Networks
}

\author{
Mawloud Omar, Sabrine Hedjaz, Souhila Rebouh, Katia Aouchar, \\ Bournane Abbache, and Abdelkamel Tari \\ Laboratoire d'Informatique Médicale, Faculté des Sciences Exactes \\ Université de Bejaia, 06000 Bejaia, Algérie.
}

\begin{abstract}
A mobile ad-hoc network is a collection of nodes forming a dynamic wireless network without pre-existing infrastructure or centralized administration. Mobile ad-hoc network is mostly used in disaster management, military applications, vehicular communication, etc. Each node in the network acts as a router and uses a routing protocol in order to transfer data in a fully distributed way. In this paper, we focus on the routing problematic in mobile ad-hoc networks and we contribute by proposing DS2R2P (onDemand Source Routing with Reduced Packets Protocol). Our proposal is based on source routing when the source node embeds the route information in the data packet allowing to drive intermediate nodes in order to reach the intended destination. Unlike existing solutions, the header of data packet includes only a reduced integer value instead of the complete sequence of intermediate node addresses. This value is considered as the summary of the routing path and includes a complete information allowing the data packet to reach the destination. We have evaluated the performances of our solution through simulations in which our protocol provides effective results in terms of data routing delay and communication overhead.
\end{abstract}

Keywords: Mobile ad-hoc network, source routing, packet size 


\section{Introduction}

The recent development of wireless communication technology and the emergence of mobile computing prompt active research work granting the access to information anywhere and at any time. Wireless networks are classified into two categories: networks with existing infrastructure and networks without infrastructure. In the first category, the network is based mainly on cellular communications. An access point acts as a router between mobile terminals. Users can move freely without loss of connectivity from an access point to another. In mobile ad-hoc networks [1], the concept of mobility is extended to all components of the network. Unlike cell-based communication networks, no central administration are available and are the mobile nodes themselves that form independently the network infrastructure.

Because of the importance of routing protocols in dynamic multi-hop networks, a lot of routing protocols have been proposed in the literature. There are some challenges that make the design of mobile ad-hoc network routing protocols a hard task. Firstly, in mobile ad-hoc networks, node mobility causes frequent topology changes and network partitions. Secondly, because of the variable and unpredictable capacity of wireless links, packet losses may happen frequently. Moreover, mobile nodes have restricted power, computing and bandwidth resources and require effective routing schemes. Such protocols should guarantee the efficient delivery of data across ad-hoc networks while maintaining a minimum communication overhead, high throughput and low end-to-end delay. The designer should consider bandwidth constraints of the wireless links, fading, interference, packet loss, exhaustible energy supply, limited computing capabilities, and a dynamic topology [5]. Existing solutions are classified mainly in two categories of protocols: proactive and reactive. In proactive routing protocols, each node in the network maintains a routing table for the broadcast of the data packets and want to establish connection to other nodes in the network. These nodes record for all the presented destinations, number of hops required to reach each destination in the routing table. To retain the stability, each node broadcasts and modifies its routing table periodically which involves a high overhead. A reactive protocol generates lower overhead since routes are determined on-demand. 
It searches for the route in an on-demand manner and set the link in order to send out and accept the packet from a source node to a destination node. Route discovery process is used by flooding the route request packets throughout the network [4]. In this paper, we focus on the category of reactive routing protocols and we contribute to this understanding by proposing DS2R2P (on-Demand Source Routing with Reduced Packets Protocol). Our proposal is based on source routing when the source node embeds the route information in the data packet allowing to drive intermediate nodes in order to reach the intended destination. Mobile nodes exchange control packets, as the request packet, the response packet and the error packet. Unlike existing solutions, the header of data packet includes only a reduced integer value instead of the complete sequence of intermediate node addresses. This value is considered as the summary of the routing path and includes the complete information allowing the data packet to reach the route destination.

The rest of this paper is organized as follows. In Section 2, we present the related work. In Section 3, we present a detailed description of our protocol. In Section 4, we present the performance analysis of our protocol. Finally, we conclude the paper in Section 5 .

\section{Related work}

Routing protocols in mobile ad-hoc networks may be categorized into three classes: proactive routing protocols, reactive routing protocols and hybrid protocols [3]. We suggest to the reader a very useful and comprehensive survey in [7]. In the literature they are a huge number of solutions of routing in mobile ad-hoc networks. In this paper, we focus on the category of reactive protocols and in grand part the solutions based on source routing, in which we present some relevant protocols. Whenever there is a need of a path from any source to the destination then a type of query reply dialog does the work. Therefore, the latency is high; however, no unnecessary control messages are required. Dynamic Source Routing protocol (DSR) [9, 11] is a source-routed on-demand routing protocol. A node maintains route caches containing the source routes that it is aware of. The route request packet contains the address of the source and the destination. Upon receiving this packet, each 
intermediate node checks whether it knows of a route to the destination. If it does not, it appends its address to the route record of the packet and forwards the packet to its neighbors. A route reply is generated when either the destination or an intermediate node with current information about the destination receives the route request packet. A route request packet reaching such a node already contains, in its route record, the sequence of hops taken from the source to this node. Ad-hoc On-demand Distance Vector Routing (AODV) [13] minimizes the number of broadcasts by creating routes on-demand. To find a path to the destination, the source broadcasts a route request packet. The neighbors in turn broadcast the packet to their neighbors till it reaches an intermediate node that has a recent route information about the destination or till it reaches the destination. When a node forwards a route request packet to its neighbors, it also records in its tables the node from which the first copy of the request came. This information is used to construct the reverse path for the route reply packet. AODV uses only symmetric links because the route reply packet follows the reverse path of the route request packet. As the route reply packet traverses back to the source, the nodes along the path enter the forward route into their tables. Location-Aided Routing (LAR) [12] proposes the use of position information to enhance the route discovery phase of reactive ad-hoc routing approaches. Reactive ad-hoc routing protocols frequently use flooding as a means of route discovery. Under the assumption that nodes have information about other node positions, this position information can be used by LAR to restrict the flooding in a certain area. Ad-hoc on-demand QoS routing based on Bandwidth Prediction (AQBP) [2] takes node future bandwidth into consideration when selects a route. The future bandwidth requirement of each node is predicted by its history. Multi Route AODV-Ant routing Algorithm (MRAA) [8] proposes two methods to improve the AODV protocol. The main goal in the design of the protocol was to reduce the routing overhead, buffer overflow and end-to-end. A multi-path routing protocol is proposed which is based on AODV and Ant Colony Optimization (ACO). Moreover, it integrates a load balancing method that uses all discovered paths simultaneously for transmitting data. In this method, data packets are balanced over discovered 
paths and energy consumption is distributed across many nodes through the network. Hash-Based Dynamic Source Routing (HB-DSR) [10] proposes to compress the list of addresses. Instead of including a source-routing option in each data packet, the source node insert the corresponding Bloom filter. HB-DSR reserves 3 bytes in the case of IPv4 and 4 bytes in the case of IPv6. HB-DSR improves DSR in terms of overhead, however the size of data packet header still remains considerable. Moreover, the problem of collision generates further overhead in terms of communication. EST-based Automatic Route Shortening (EST-ARS) [6] introduces the so-called Expected Sending Times (EST) to evaluate the quality of links, which is used to perform an automatic route shortening process. The EST of a link is considered as the expected number of data transmissions required to send a packet over that link. The EST of a route is the sum of the EST for each link in the route. The calculation of links EST is performed using hello packets, and a low value of EST means that it consumes less time and energy to send a packet successfully, and vice versa. Obviously, the smaller EST of a link is, the better the link quality. After a node outside source route overheard a data packet, if it finds that there is a shorter route to the destination node of the packet in its own route cache, it should return a request of routing comparison, which gives the full path to the original node. Then, the source node will launch the comparison process about the values of EST of the original route and the candidate route.

\section{Our protocol}

In this section, we present our routing protocol entitled DS2R2P (onDemand Source Routing with Reduced Packets Protocol). We model the considered environment and then, we give a detailed description of the elements composing DS2R2P.

\subsection{Network model}

We consider mobile ad-hoc networks where a set of $\partial$ mobile nodes are randomly located in a two-dimensional space and move with a random mobility pattern, i.e., nodes moving independently. Each node $v_{i}$ has a single 
channel and hence, we will use the same identifier for the node and its address. The network topology changes frequently due to the mobility of nodes. We assume that mobile nodes have the same transmission power range $\rho$. The set of nodes in the $v_{i}$ 's vicinity denoted by $\Theta_{i}$, consists of $v_{i}$ 's neighboring nodes, defined as: $\Theta_{i}=\left\{v_{j}: d_{\left(v_{i}, v_{j}\right)} \leq \rho\right\}$, where $d_{\left(v_{i}, v_{j}\right)}$ denotes the Euclidean distance between nodes $v_{i}$ and $v_{j}$. We note that the communication links are bidirectional, i.e., if $v_{j} \in \Theta_{i}$ then, $v_{i} \in \Theta_{j}$. The network can be modeled by a graph $G=(V, E)$, where $V$ is the set of all nodes and $E$ is the set of all direct links. There is an edge between two nodes if they are located within each other transmission range. A path between the source node $v_{S}$ and the destination node $v_{D}$ can be represented as a node sequence $\mathrm{R}=\left(v_{\mathrm{S}}, \ldots, v_{i}, \ldots, v_{\mathrm{D}}\right)$, where $v_{i} \in \mathrm{V}$.

\subsection{Overview of DS2R2P}

DS2R2P operates in two main phases: route discovery and data packet routing. Route discovery is started before transmitting a data packet if the node $v_{S}$ cannot find a routing path in its cache reaching the destination node $v_{D}$. This step is executed in a diffusive manner using a route request packet (RREQ) looking for available routing paths which connect nodes $v_{S}$ and $v_{\mathrm{D}}$. Upon receiving the RREQ packet, the node $v_{\mathrm{D}}$ responds with a route reply packet (RREP) including the complete routing path using the reverse way. Each intermediate node summarizes the sub-route that connect it to $v_{\mathrm{D}}$ and stores the summary in its cache. When the source node needs to send data to its interlocutor under the routing path, how the latter is used in order to transmit the data packet to the destination node? Instead of attaching the routing path to the request packet (classical source-routing), we introduce a mechanism which provides a single integer value considered as the route summary. Each intermediate node uses the received summary in order to deduce its successor, and so on, until the destination node is reached. DS2R2P operates using caches unlike existing reactive routing protocols. Indeed, in proactive protocols, a node checks periodically the state of links and updates routes in its cache by maintaining only the set of valid ones. This process produces a considerable overhead given the large number of generated messages of control. However, in DS2R2P, link management is 
not executed periodically. The node keeps temporary and only the lasts active routes with indexes, discovered in the last route discovery process. Broken links are detected only when routing a data packet, and in this case, the intermediate node drops all the indexed sub-routes that pass through the broken link. Then, a route error packet (RRER) will be returned to the source node and the latter initiates the route discovery process.

\subsection{Route discovery}

Each node in the network maintains in its locally a cache keeping the routes track of this node toward different destination nodes in the network. This table is indexed by summaries of stored sub-routes. Each line in this table consists of two fields: the index which represents the result obtained by reducing the routing path; and the sub-route in its original format. When a node requires to send data, firstly it checks if a route is already stored in its cache. In the opposite case, the node broadcasts a request packet RREQ in the network in order to gather information relating to available routes to reach the destination. Upon receiving the RREQ packet, each intermediate node includes its own address and forward it to its neighbor nodes. This process is repeated until the RREQ packet reaches the destination node. Finally, the latter builds-up the reply packet RREP and computes the summary of its own address $\Omega\left(v_{D}\right)$. The latter and the corresponding sub-route will be stored in its cache, where the sub-route includes initially just the destination node address. The function of reduction is defined as follows:

$$
\Omega\left(v_{1}, v_{2}, \ldots, v_{n}\right)=\left[\sum_{i=1}^{n} v_{i} \times c^{i \times e}\right] \bmod \eta
$$

where $v_{i}$ denotes the set of intermediate node identities and $n$ denotes the route length. $\eta$ is a prime integer allowing to summarize the routing path $R=\left(v_{1}, v_{2}, \ldots, v_{n}\right)$ to an integer value representing the index of the routing path. The generated summary (the function $\Omega$ 's output) is strictly inferior to the value of $\eta$, which allows both to reduce and fix the size of the index. Whatever the length of the route $R$, the size of the index will be in the order of $\log _{2}(\eta)$ bits. $c$ and $e$ are a large integers, introduced in order to disperse 
the space of possible values of the function $\Omega$, which reduces the probability of collisions. The factor $i$ is introduced in order to produce different outputs of $\Omega$ in the case of routing paths composing by the same intermediate nodes in a different order.

The function $\Omega$ can be considered as a particular hash function. Existing hash mechanisms are computationally expensive and their use in lower layers of the protocols stack (network layer) decreases the performance of the routing protocol. The function $\Omega$ may produce collisions and its effectiveness is strongly depending on the network size. When the network size increases significantly, the nodes addressing range increases, which can cause collisions. The probability to get the same summary corresponding to two different routing paths is negligible. This is due to the fact that the function $\Omega$ combines the inputs (the identities of intermediate nodes) which are independent parameters unlike conventional hashing functions, that transform a high data block into a reduced value size which increases the possibility of collisions.

The destination node sends the RREP packet using the reverse way of the gathered route within the RREQ packet. Upon receiving the latter, each intermediate node adds its own address to the sub-route, computes the summary of the novel sub-route and stores both parts in its cache. This process is repeated until the RREP packet reaches the source node. The process of route discovery is illustrated in figure 1 .

\subsection{Data packet routing}

Let's consider a route $R=\left(v_{1}, v_{2}, \ldots, v_{n}\right)$, where $v_{1}=v_{S}$ and $v_{n}=v_{D}$ denote respectively the source and destination nodes. Following classical source-routing techniques, the source node includes to the request packet the complete route (the list of intermediate node addresses of the routing path). Upon receiving this packet, each intermediate node memorizes its predecessor (the identifier of the node from which it receives the packet), checks the list of intermediate nodes, erases its identifier from this list, and forwards the packet to the next one. This process is repeated until the packet reaches the destination node, i.e., until the route length equals to 1 . This solution is unsuitable for mobile networks in large scale regarding to the 


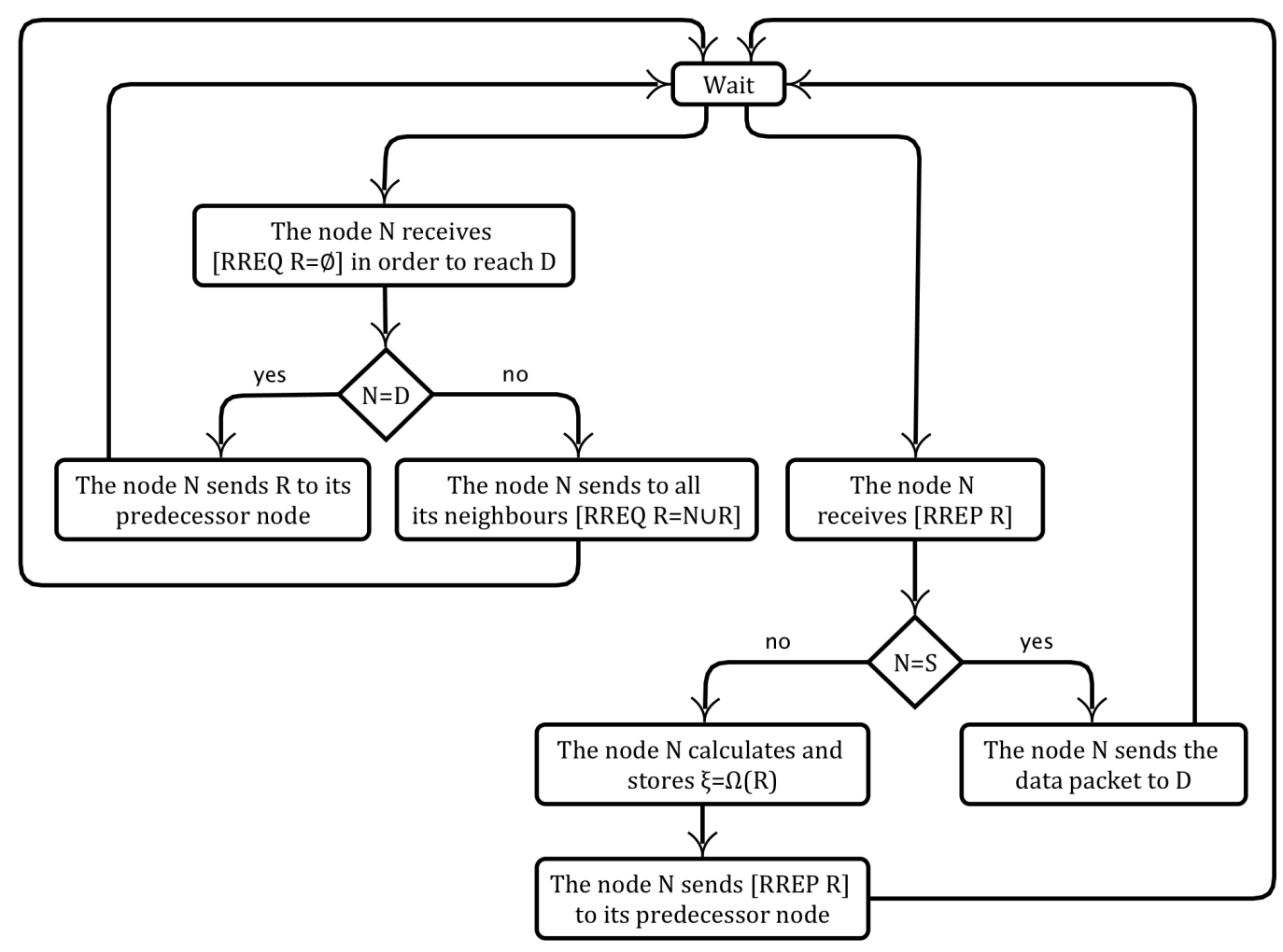

Figure 1: Route request and reply packets routing in DS2R2P

high longer paths which can be considered. To overcome this problem, our protocol proceeds as follows. Instead of attaching the compete routing path, the source node $v_{S}$ computes and embeds $\xi_{S}=\Omega\left(v_{2}, v_{3}, \ldots, v_{n}\right)$ in the data packet and sends it to the first intermediate node $v_{2}$. It should be noted that whatever the size of the routing path, the summary is strictly lesser than $\eta$. Comparing to DSR, which operates with $32 \times n$ bits of header size, DS2R2P reduces this value to $\log _{2}(\eta)$ bits. Upon receiving the data packet, each intermediate node $v_{i}$ looks in its cache for the sub-route which is indexed by the received summary. Then it computes $\xi_{i}=\Omega\left(v_{i+1}, v_{i+2}, \ldots, v_{n}\right)$, replaces the old summary value with the new one in the data packet and sends the latter to the node $v_{i+1}$. This process is performed end-to-end by the set of intermediate nodes until the data packet reaches the destination node 
$v_{\mathrm{D}}=v_{n}$

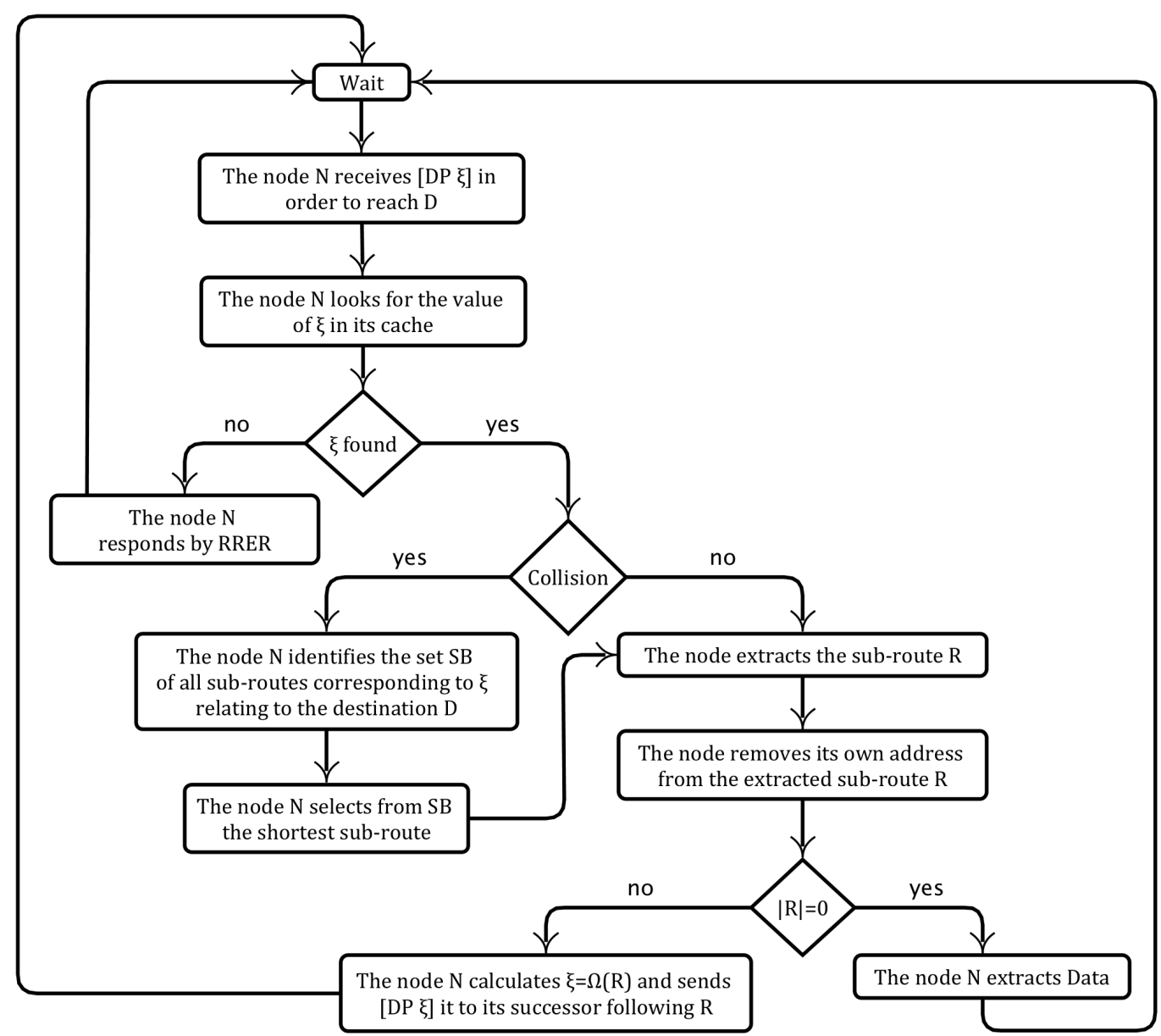

Figure 2: Data packets routing in DS2R2P

Seldom possible, a node can find in its cache several sub-routes that correspond to the same value of the received summary which can be caused by collisions through the reduction function. In this case, the node selects the shortest sub-route among those corresponding to the summary with condition that the end of the selected sub-route should be the intended destination node which is indicated in the received data packet. It should be noted that whatever the number of sub-routes corresponding to the summary, only 
one sub-route will be used without any additional load in terms of control messages. The process of data packet routing is illustrated in figure 2.

As a link in a routing path can be broken due to mobility, fluctuations of communication channels or due to nodes failures, it is necessarily to update the routes. If an intermediate node detects a broken link when forwarding a specific data packet, it sends a RERR packet to the source node. An intermediate node, upon receiving the RERR packet, it deletes from its cache all entries that use this link. If the source node does not have any valid route in its cache, it initiates the route discovery process.

\subsection{Practical characteristics of DS2R2P}

We analyze the practical adaptation of DS2R2P by involving the required hardware performances. Three criteria are addressed: the storage, the communication overhead and the transmission time. We refer often to the basic DSR protocol in order to illustrate practical improvements. It should be noted that DSR is involved just to draw illustrations, and the performance evaluation of our protocol is discussed in Section 4 by comparison with another protocol.

\subsubsection{Storage requirement}

Each mobile node $v_{i}$ maintains a table in which each line includes the index and the corresponding sub-route. Therefore, is allocated to the first field $\log _{2}(\eta)$ bits. We consider the worst case in which each node maintains routing paths composed of all nodes of the network. In this case, $32 \partial$ bits are allocated for the second field (we consider the case of IPv4 in which nodes addressed are in 32 bits). The number of lines in the cache depends on the number of possible routes that pass through the node in question. We consider the worst case in which the node is an intermediate node connecting each pair of nodes in a fully connected network. With a network size of $\partial$ nodes, the number of possible routes connecting each pair of nodes equals to $\frac{\partial}{2}(\partial-1)$. Thus, the required capacity of storage memory $\mathcal{M}$ can be calculated such as:

$$
\mathcal{M}=\frac{\partial(\partial-1)\left(\log _{2}(\eta)+32 \partial\right)}{2}
$$




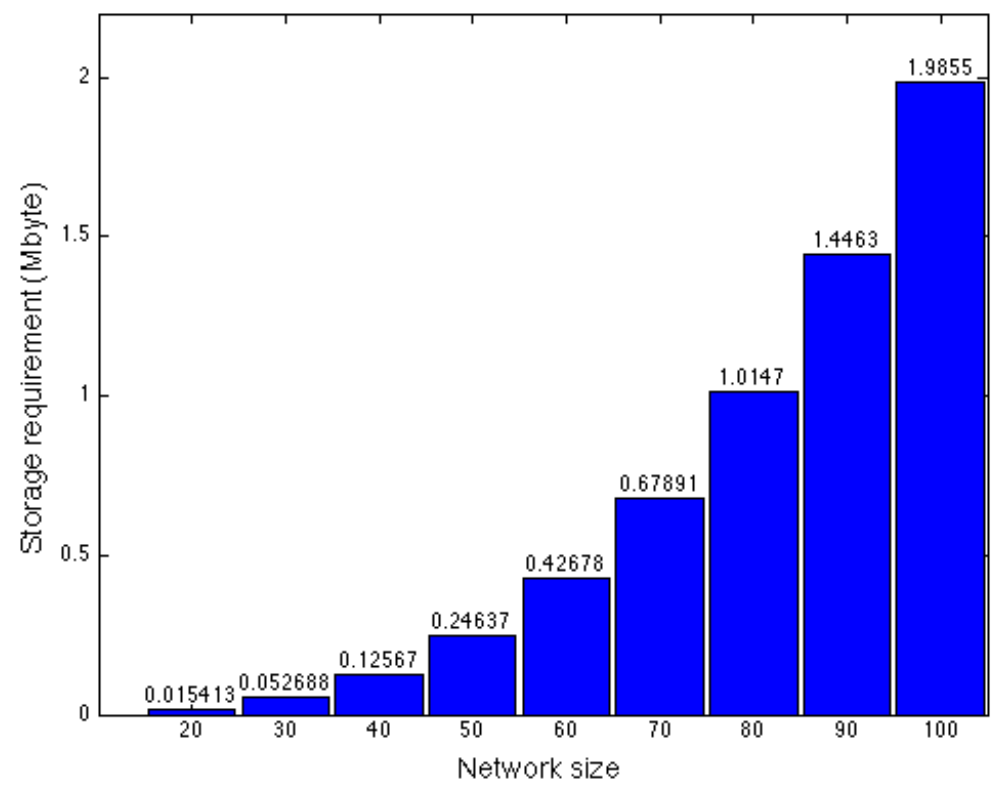

Figure 3: Storage requirements in function of network size

Figure 3 illustrates the maximal storage capacity required per node in function of various network sizes with $\eta=499$. For example, with a network size of 500 nodes, each node reserves in the worst case approximately 2 Mbyte. Therefore, we note that DS2R2P does not present hard constraints in terms of storage capacity requirements according to the technology we have today. We note that in terms of storage, our protocol requires the same capacity as in proactive routing algorithms. However, the latters spend much bandwidth due to the periodic exchange of control messages in order to maintain valid routing paths in the cache.

\subsubsection{Transmission delay}

Let's consider a route $R=\left(v_{1}, v_{2}, \ldots, v_{n}\right)$, where $v_{1}=v_{S}$ and $v_{n}=v_{D}$. We assume that all nodes have the same hardware characteristics and the same wireless interface debit $\mathrm{Db}$. We assume that the traffic in the network is light, and hence the communication channel is assumed to be error free 
and provides reliable data delivery. We consider a scenario in which the source node $v_{S}$ generates and sends a data packet $P_{0}$ under the route $R$. The data packet travels the routing path under each intermediate node $v_{i}$ until to reach the destination $v_{D}$. Each intermediate node $v_{i}$ upon receiving $P_{i-1}$ from the node $v_{i-1}$, it updates the packet by adjusting the $P_{i-1}$ 's header field and forwards the packet $P_{i}$ with new header parameters to the node $v_{i+1}$. The data packet $P_{i}$ is composed of two parts: the data, denoted by $\vartheta$, and the packet header, denoted by $\Delta_{i}$. Thus, the size of the data packet $\mathrm{P}_{i}$ can be calculated by $\left|P_{i}\right|=|\vartheta|+\left|\Delta_{i}\right|$, and the time of transmission of the packet from $v_{i}$ to $v_{i+1}$ can be calculated such as:

$$
\mathrm{T}_{i}=\frac{|\vartheta|+\left|\Delta_{i}\right|}{\mathrm{Db}}
$$

Following the basic protocol of DSR, each intermediate node $v_{i}$ removes its own address from the header and forwards the data packet to its successor node. Thus, at each hop the header size is decremented by 32 bits $\left(\left|\Delta_{i}\right|=\right.$ $\left.\left|\Delta_{i-1}\right|-32\right)$. The transmission time $\mathcal{T}_{\text {DSR }}$ of the data delivery toward the destination node $v_{D}$ is the sum of transmission times $T_{i}$ per each intermediate node $v_{i}$ :

$$
\mathcal{T}_{\text {DSR }}=\sum_{i=1}^{n-1} \frac{|\vartheta|+\left|\Delta_{0}\right|-i \times \frac{\left|\Delta_{0}\right|}{n}}{\mathrm{Db}}
$$

We note that $\left|\Delta_{0}\right|=32 \times(n-1)$, where $n$ is the number of nodes composing the full routing path and $n-1$ is the number of hops. Thus,

$$
\mathcal{T}_{\mathrm{DSR}}=\frac{(\mathrm{n}-1)|\vartheta|+16(\mathrm{n}-1)(\mathrm{n}-2)}{\mathrm{Db}}
$$

Following DS2R2P, each intermediate node $v_{i}$ upon receiving the data packet, replaces the current summary $\Delta_{i-1}$ in the header with $\Delta_{i}$, calculated by the node $v_{i}$. The transmission time $\mathcal{T}_{\text {DS2R2P }}$ of data delivery to the destination node is the sum of transmission times $T_{i}$ per each intermediate node 
$v_{i}:$

$$
\mathcal{T}_{\text {DS2R2P }}=\sum_{i=1}^{n-1} \frac{|\vartheta|+\left|\Delta_{i}\right|}{\mathrm{Db}}
$$

Whatever the size of the routing path, the function of reduction outputs a result strictly lesser than $\eta$. Thus, in all cases $\left|\Delta_{i}\right|=\log _{2}(\eta)$, and hence:

$$
\mathcal{T}_{\text {DS2R2P }}=\frac{(n-1)\left(|\vartheta|+\log _{2}(\eta)\right)}{\mathrm{Db}}
$$

Figure 4 and 5 illustrate, respectively, following the protocol DSR and DS2R2P, the transmission delay per data packet in function of various routing path lengths. We have considered three norms of wireless technology: (1) the norms 802.11a and 802.11g with a debit of 11 Mbps, (2) the norm 802.11b with a debit of $54 \mathrm{Mbps}$ and (3) the norm 802.11n with a debit of $540 \mathrm{Mbps}$. We note that DS2R2P with a debit of 11 Mbps achieves approximately the same performances in terms of transmission delay compared to the protocol DSR with a debit of 54 Mbps. The length of the routing path denotes the number of intermediate nodes composing the routing path. The impact of this parameter on DSR protocol is significant in which the source node embeds the routing path in the packet header. Hence, the route length influences strongly the packet size and accordingly increases the transmission delay of the packet.

\subsubsection{Communication overhead}

We express by the communication overhead the quantity of required information of control which should be embedded in the data packet in order to drive the latter to the final destination. Information of control are not part of the data user, however it should be reduced in order to reduce the congestion in the network. Following the protocol DSR, intermediate nodes generate an overhead of $\mathcal{O}=16(n-1)(n-2)$ bits per data packet. However, in DS2R2P, they generate $\mathcal{O}=(n-1) \log _{2}(\eta)$ bits.

Figure6 illustrates a comparison between the protocol DSR and DS2R2P in terms of the communication overhead per data packet in function of various routing path lengths. The gap in terms of communication overhead is largely 


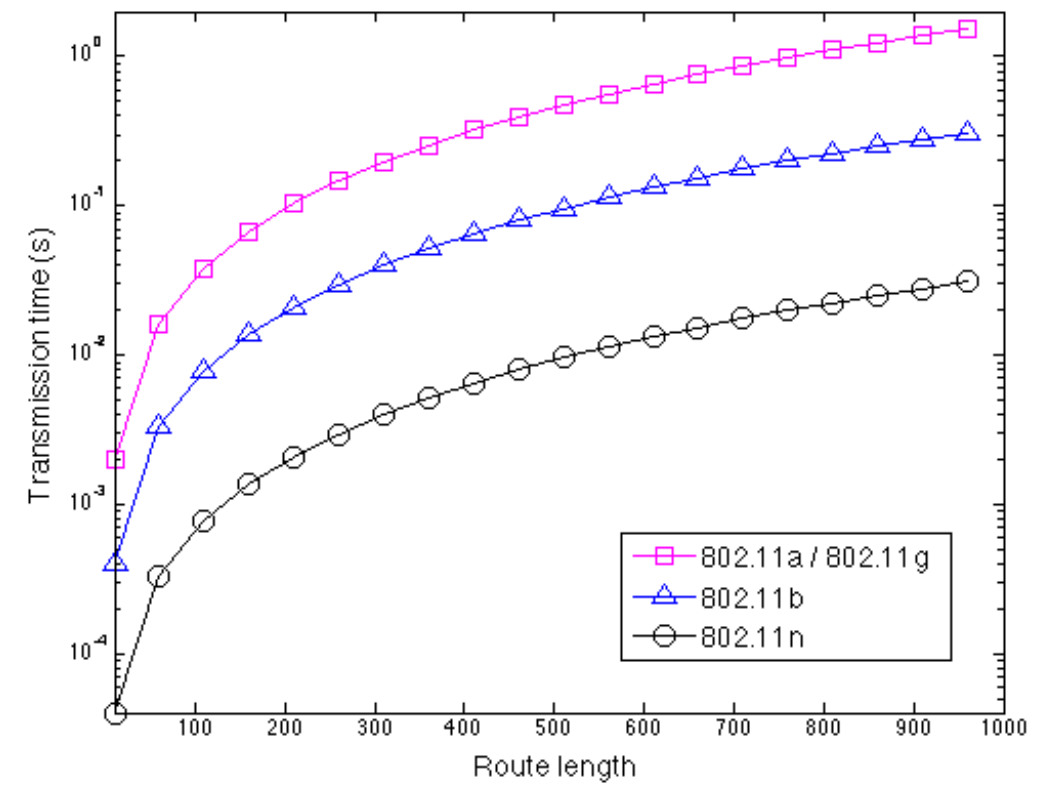

Figure 4: Transmission time per data packet in function of routing path lengths following the protocol DSR $(|\vartheta|=256$ byte $)$

great due to the difference between the header size of the data packet which is reduced in DS2R2P to $\log _{2}(\eta)$ bits.

\section{Simulation results}

In this section, we present the parameters and assumptions related to the environment of simulation, and then we present and discuss the obtained results.

\subsection{Parameters and assumptions}

We have implemented our simulations using Matlab environment [14]. We have simulated a mobile ad-hoc network up to 500 nodes moving in a square area of $1 \mathrm{~km}^{2}$. We have considered two cases of nominal range: a normal radio propagation mode, which is varied uniformly in the interval of $\rho \in[125 \mathrm{~m}, 200 \mathrm{~m}]$, and a high radio propagation mode, which is varied 


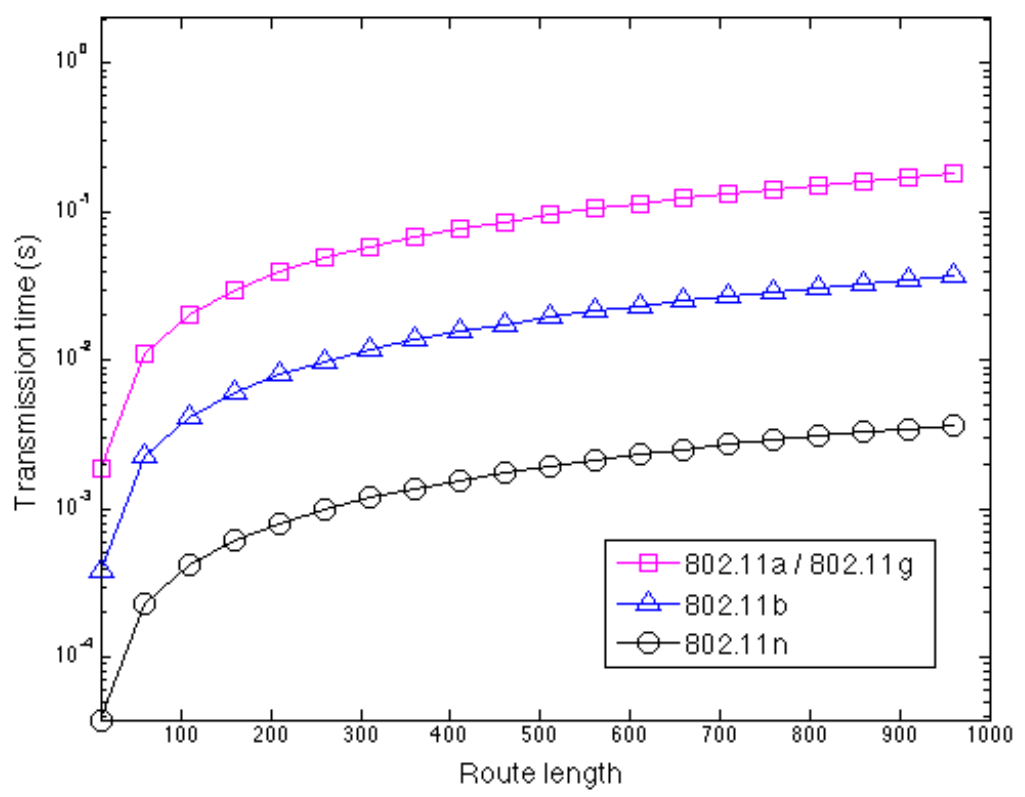

Figure 5: Transmission time per data packet in function of routing path lengths following the protocol DS2R2P $(\eta=499$ and $|\vartheta|=256$ byte $)$

uniformly in the interval of $\rho \in[350 \mathrm{~m}, 400 \mathrm{~m}]$. Each node is configured with a wireless device with a transmission speed of 54 Mbps. The movement pattern is defined by the random waypoint model. A mobile node moves in the area from its current position to a new location by randomly choosing a destination coordinates and the time that it will pause. After the pause time, the node chooses a new destination and pause time. This is repeated for each node, until the end of the simulation time. Our simulator estimates if a radio link exists among nodes according to the distance that separates them. We assume that nodes have the same hardware characteristics and processing capabilities. We have compared DS2R2P to the protocols DSR [9, 11] and EST-ARS [6]. We have studied the impact of network size in order to evaluate the scalability by considering three metrics of performance:

1. The transmission delay which represents the average delay from the generation of a data packet until to its reception by the destination. 


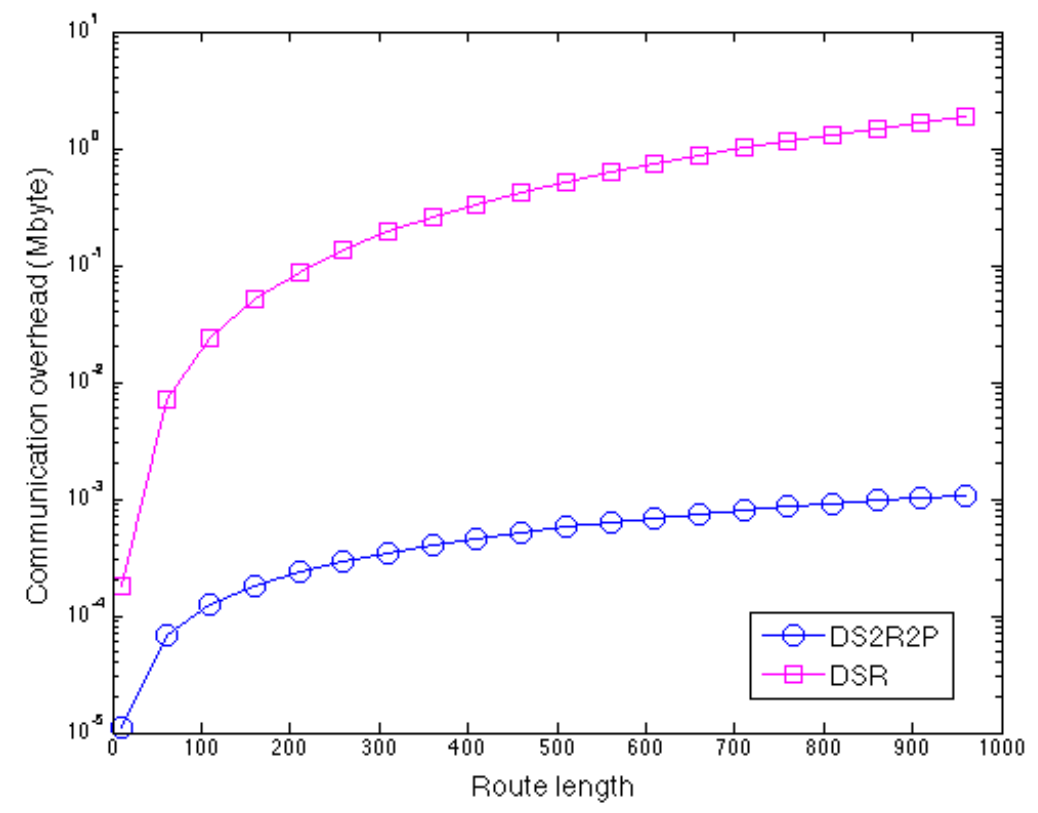

Figure 6: Data packet header size in function of routing path lengths $(\eta=499)$

2. The communication overhead which represents the total size of exchanged packets in the network.

3. The efficiency which represents the ratio of data packet size versus the user data.

\subsection{Results of comparison}

In figures 7 and 8, we illustrate the results of comparison in terms of transmission time in function of the network size, respectively in the case of normal and high nominal range. Transmission delay represents the average delay from the generation of the data packet until to its reception by the destination. When the network size increases, the network becomes more dense which increases the number of available routing paths between each pair of nodes in the network. The gap in terms of transmission time between DS2R2P and the other protocols is due to the large size of routing paths 


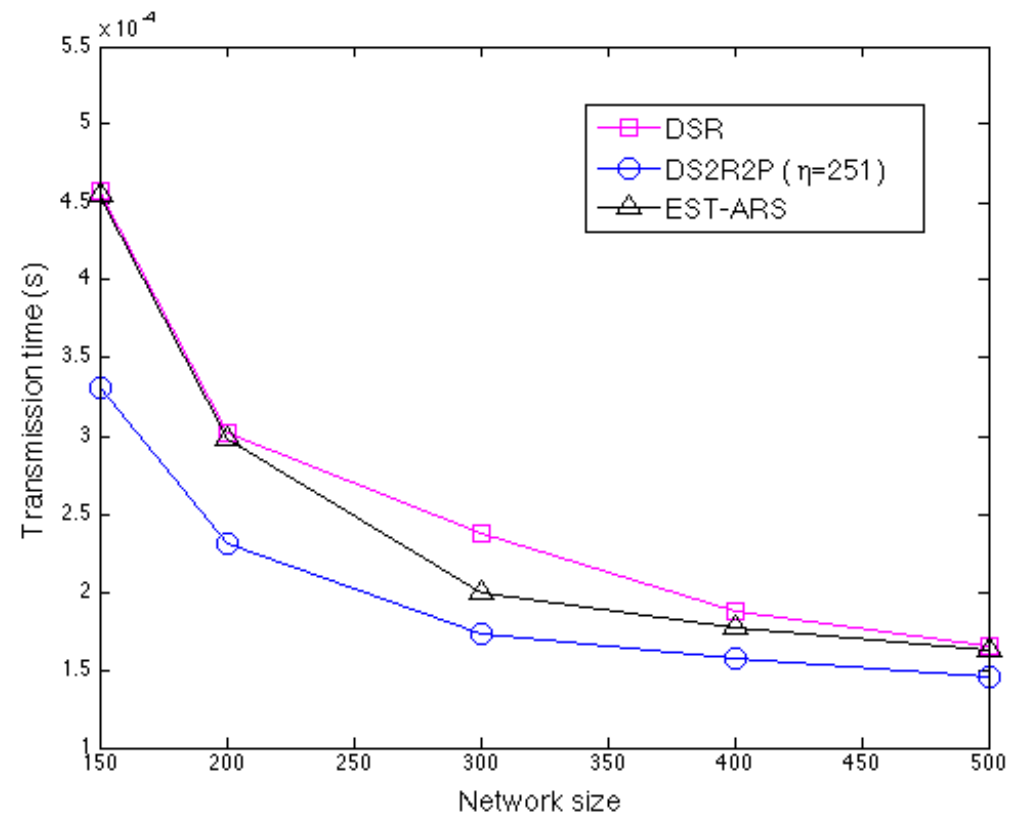

Figure 7: Transmission time in function of the network size with normal propagation range

embedded in the data packets. The size of data packets in DS2R2P is reduced which allows nodes to reach rapidly their interlocutors. Although the routing path of EST-ARS which is more shorter, this do not involve a significant reduction in terms of transmission time with comparison to DS2R2P.

In figures 9 and 10 , we illustrate the results of comparison in terms of communication overhead in function of the network size, respectively in the case of normal and high nominal range. The communication overhead represents the total size of exchanging packets in the network. The protocols DSR and EST-ARS embed the complete sequence of the routing path in data packets, which increases the communication overhead in the network. In DS2R2P, the data packet header includes a fixed and reduced value of $\log _{2}(\eta)$ bits which interprets the gap in terms of communication overhead with comparison to the other protocols. It should be noted that although the size of the header size is little it includes the complete information allowing the source node to reach its interlocutor. 


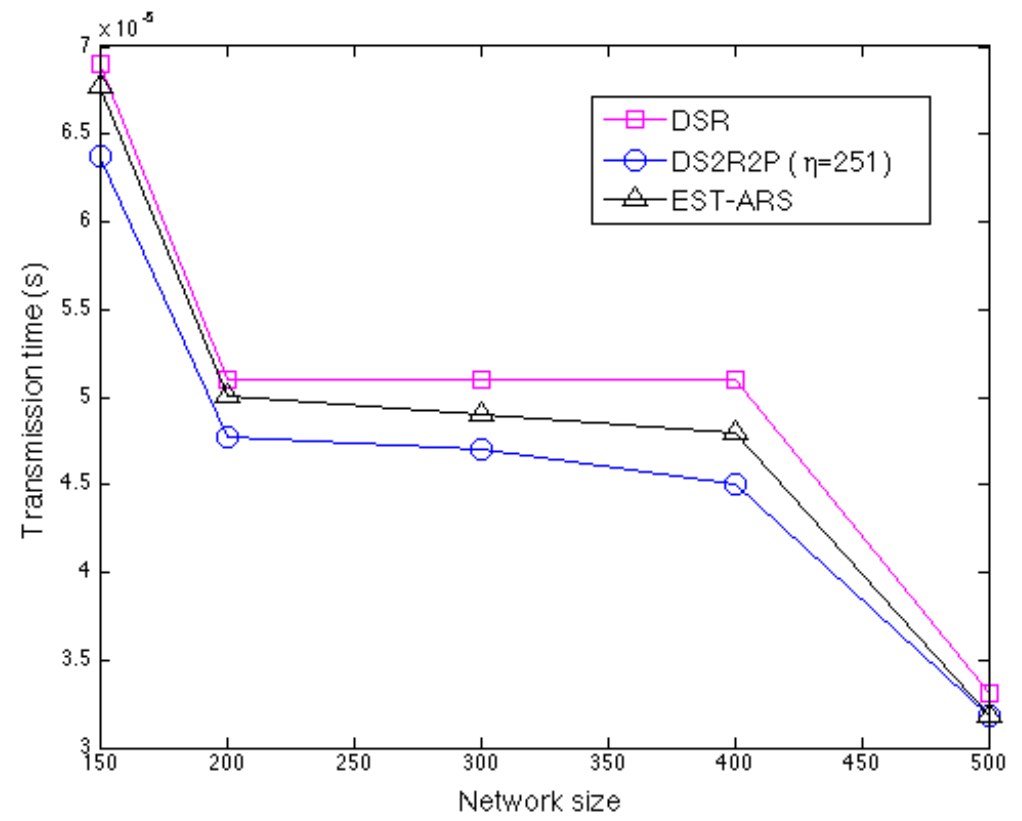

Figure 8: Transmission time in function of the network size with high propagation range

In figures 11 and 12, we illustrate the results of comparison in terms of efficiency in function of the network size, respectively in the case of normal and high nominal range. The efficiency is calculated as follows:

$$
\mathrm{E}=\frac{|\vartheta|}{|\vartheta|+|\Delta|}
$$

which represents the proportion of spared header size with respect to the total size of the data packet. DS2R2P achieves the best results, which is due to the reduced size of packet header that equals to:

$$
\mathrm{E}_{\mathrm{DS} 2 \mathrm{R} 2 \mathrm{P}}=\frac{|\vartheta|}{|\vartheta|+\log _{2}(\eta)}
$$

It should be noted that each time the size of the routing path is reduced, the ratio efficiency of the three protocols is increasing. This can be interpreted by the fact that nodes can find shorter routing paths due to the large 


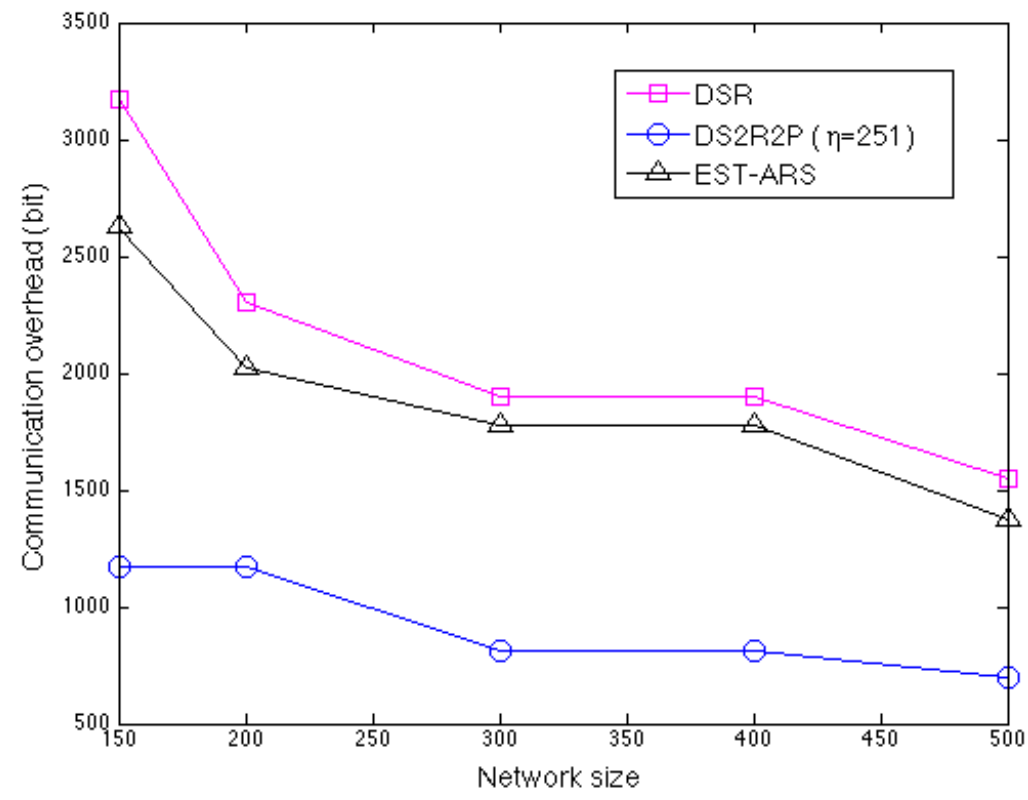

Figure 9: Communication overhead in function of network size with normal propagation range

number of available routes.

\section{Conclusion}

In techniques of source routing, the communication load is very high when the source node embeds the complete sequence of intermediate node addresses in the header field of the data packet. In order to overcome this problem, we have proposed DS2R2P which improves routing performances in terms both transmission time and communication overhead. Our protocol summarizes the routing path into a reduced integer value and each intermediate node can deduce its successor. We have evaluated the performances of our solution through simulations in which our protocol provides interesting results. 


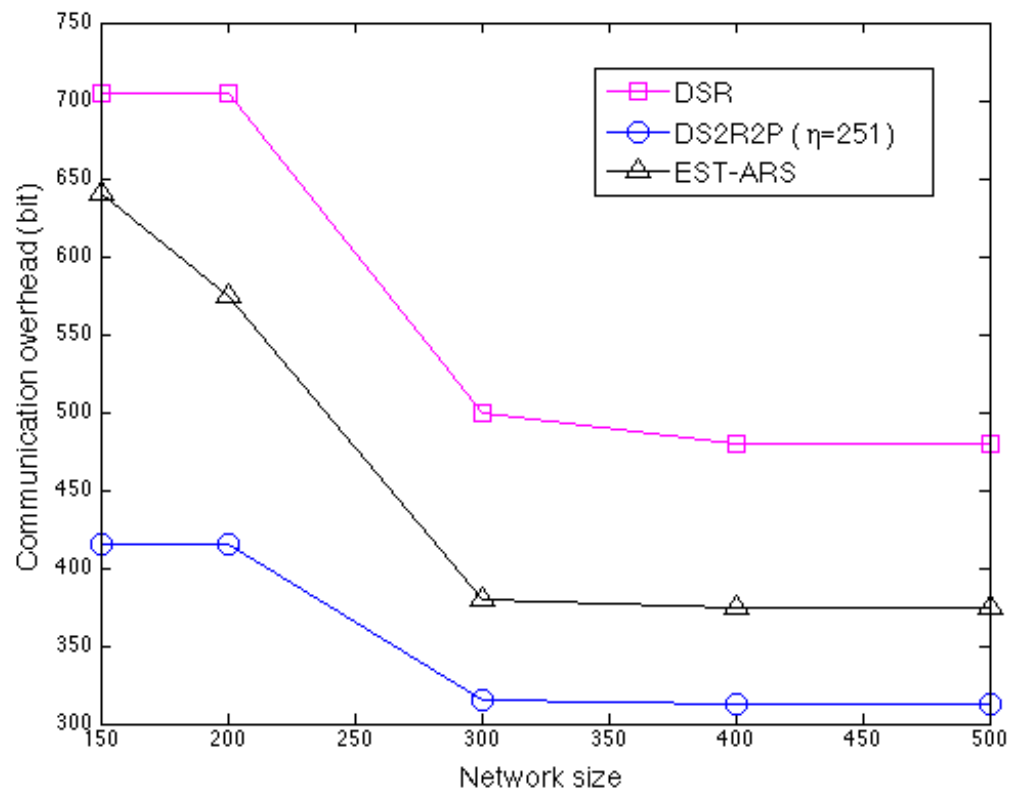

Figure 10: Communication overhead in function of the network size with high propagation range

\section{References}

[1] Basagni S., Conti M., Giordano S., Stojmenovic I. Mobile ad hoc networking: the cutting edge directions. John Wiley and Sons Inc., Hoboken, New Jersey, 2013.

[2] Liu L., Zhou J. Ad hoc on-demand QoS routing based on bandwidth prediction. In proceedings of the 8th International Conference on Wireless Communications, Networking and Mobile Computing, 2012.

[3] Norouzi A., Sertbas A. Efficient analysis and comparative performance evaluation of routing protocols in mobile ad hoc network. Studies in Informatics and Control, 2012.

[4] Shivahare B-D., Wahi C., Shivhare S. Comparison of proactive and reactive routing protocols in mobile ad hoc network using routing protocol property. International Journal of Emerging Technology and Advanced Engineering, 2012. 


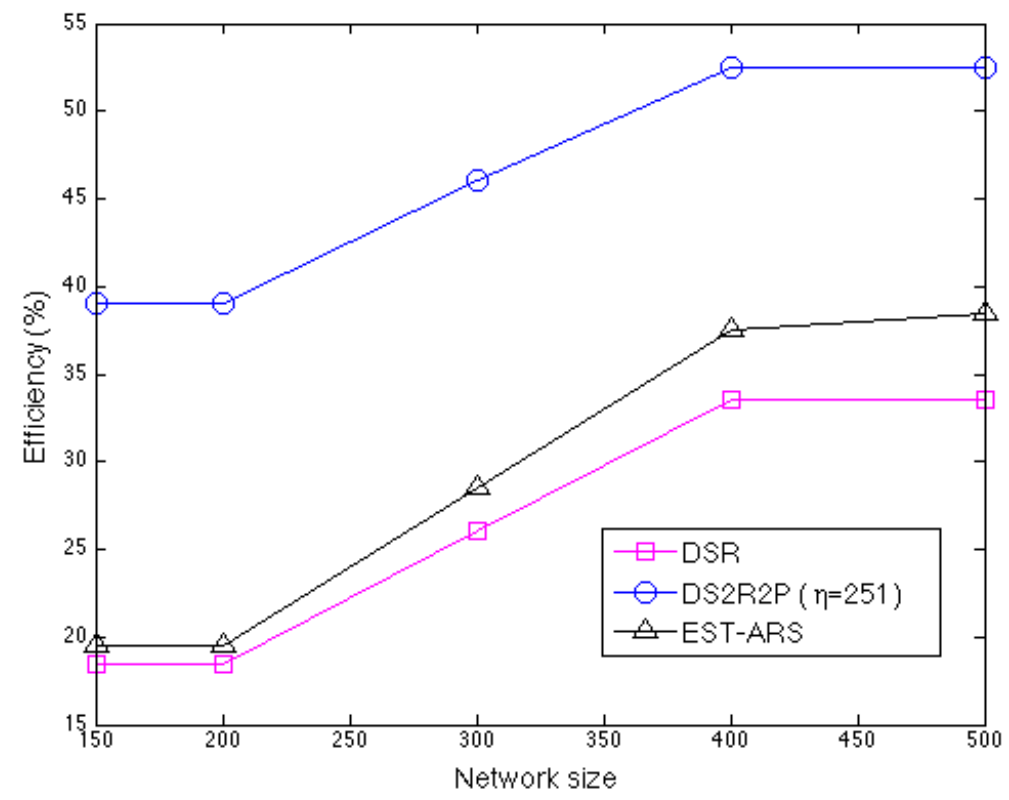

Figure 11: Efficiency in function of the network size with normal propagation range

[5] Hawa M., Taifour S., Qasem M., Tuffaha W. A dynamic cross-layer routing protocol for mobile ad hoc networks. International Journal of Electronics and Communications (AEU), 2012.

[6] Xia L., Jiang S., Song Z., Sun G. An EST-based automatic route shortening in dynamic source routing protocol. Information and Automation Communications in Computer and Information Science (Springer), 2011.

[7] Boukerche A., Turgut B., Aydin N., Ahmad M-Z., Boloni L., Turgut D. Routing protocols in ad hoc networks: a survey. International Journal of Computer Networks, 2011.

[8] AbdelMoniem M-A., Mohamed H-M., Hedar A. An ant colony optimization algorithm for the mobile ad hoc network routing problem based on AODV protocol. In the proceedings of the 10th International Conference on Intelligent Systems Design and Applications, 2010. 


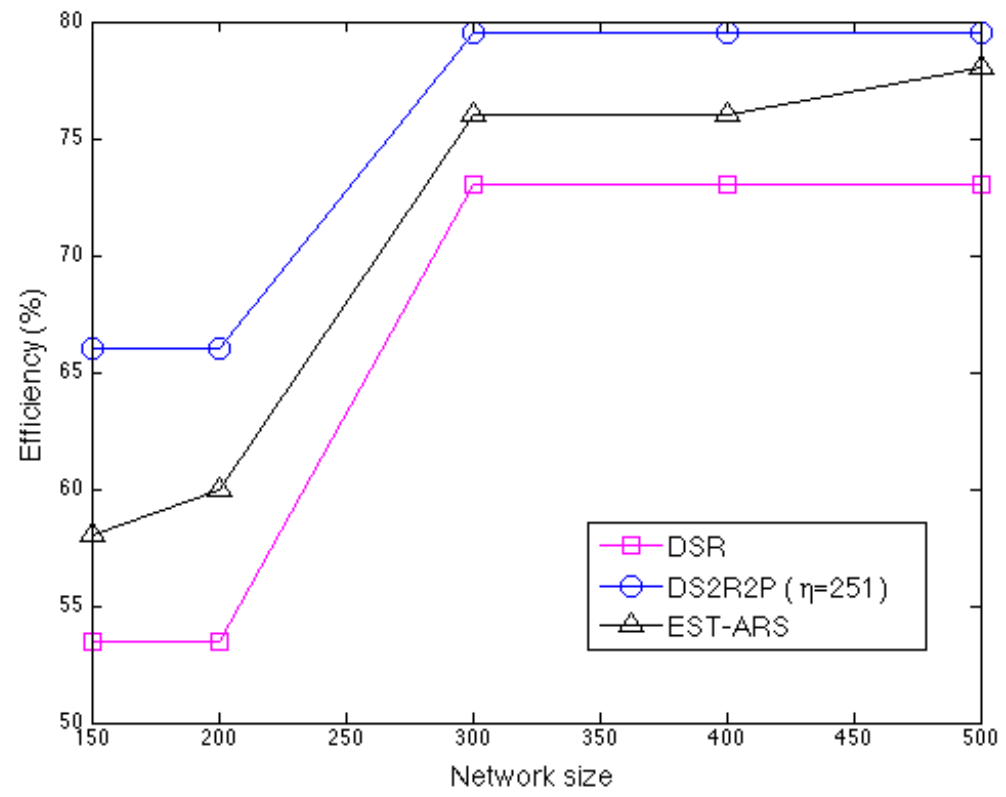

Figure 12: Efficiency in function of the network size with high propagation range

[9] Johnson D-B., Hu Y., Maltz D-A. The dynamic source routing protocol for mobile ad hoc networks for IPv4. RFC 4728, 2007.

[10] Castelluccia C., Mutaf P. Hash-based dynamic source routing. Lecture Notes in Computer Science Volume (Springer), 2004.

[11] Johnson D-B., Maltz D-A., Broch J. DSR: the dynamic source routing protocol for multi-hop wireless ad hoc networks. In Chapter 5 of ad hoc networking, edited by Charles E. Perkins, 2001.

[12] Ko Y-B., Vaidya N-H. Location-aided routing in mobile ad hoc networks. International Journal of Wireless Networks, 2000.

[13] Perkins C-E., Royer E-M. Ad-hoc on-demand distance vector routing. In proceedings of the Second IEEE Workshop on Mobile Computing Systems and Applications, 1999.

[14] http://www.mathworks.com/. 